\title{
VARIATION OF GEODESIC LENGTH FUNCTIONS IN FAMILIES OF KÄHLER-EINSTEIN MANIFOLDS AND APPLICATIONS TO TEICHMÜLLER SPACE
}

\author{
Reynir Axelsson and Georg Schumacher \\ Háskóli Îslands, Department of Mathematics \\ Dunhaga 5, IS-107 Reykjavík, Ísland; reynir@raunvis.hi.is \\ Philipps-Universität Marburg, Fachbereich Mathematik und Informatik \\ Lahnberge, D-35032 Marburg, Germany; schumac@mathematik.uni-marburg.de
}

\begin{abstract}
In the study of Teichmüller spaces the second variation of the logarithm of the geodesic length function plays a central role. So far, it was accessible only in a rather indirect way. We treat the problem directly in the more general framework of the deformation theory of Kähler-Einstein manifolds. For the first variation we arrive at a surprisingly simple formula, which only depends on harmonic Kodaira-Spencer forms. We also compute the second variation in the general case and then apply the result to families of Riemann surfaces. Again we obtain a simple formula depending only on the harmonic Beltrami differentials. As a consequence a new proof for the plurisubharmonicity of the geodesic length function on Teichmüller space and its logarithm together with upper estimates follow. The results also apply to the previously not known cases of Teichmüller spaces of weighted punctured Riemann surfaces, where the methods of Kleinian groups are not available. We use our methods from [A-S], where the result was announced.
\end{abstract}

\section{Introduction}

In the study of Teichmüller spaces geodesic length functions play an important role, in particular under the aspect of the theory of several complex variables.

In $[\mathrm{K}]$ Kerckhoff showed that for a finite number of closed geodesics, which fill up a Riemann surface, the sum of the geodesic length functions provides a proper exhaustion of the corresponding Teichmüller space. In [WO3] Wolpert proved that this function is actually convex along Weil-Petersson geodesics and plurisubharmonic. Later it turned out that the logarithm of a sum of geodesic length functions is plurisubharmonic as well [WO4, WO5]. In [Ye] Yeung constructed a bounded plurisubharmonic exhaustion function together with estimates. The Levi form of the geodesic length functions also played an important role in McMullen's proof of the Kähler hyperbolicity of the moduli space $[\mathrm{M}]$.

We want to base our study of geodesic length functions solely upon the hyperbolic geometry of Riemann surfaces and use the methods of Kähler geometry. From this point of view it is desirable to express results in terms of harmonic Beltrami differentials, which are to be considered as harmonic Kodaira-Spencer forms.

This approach avoids entirely methods involving Fuchsian groups. In particular our results extend to cases where uniformization theory is not available, such as

doi:10.5186/aasfm.2012.3703

2010 Mathematics Subject Classification: Primary 53C55, 32G15, 32Q20.

Key words: Kähler-Einstein metrics, Teichmüller theory, geodesic length functions. 
Teichmüller and moduli spaces of weighted punctured Riemann surfaces, equipped with conical hyperbolic metrics.

Infinitesimal deformations, i.e. complex tangent vectors of Teichmüller space will be represented by harmonic Beltrami differentials on hyperbolic Riemann surfaces. For Kähler-Einstein manifolds $X$ of constant negative Ricci curvature these correspond to harmonic Kodaira-Spencer forms representing elements of $H^{1}\left(X, \mathcal{T}_{X}\right)$.

Theorem 1.1. [A-S, Theorem 3.2] Given a holomorphic family $\mathcal{X} \rightarrow S$ of Kähler-Einstein manifolds with negative Ricci curvature, the first variation of the length in a family of closed geodesics $\gamma_{s}$ is a geodesic integral of the harmonic KodairaSpencer form

$$
A_{i}=A_{i \bar{\beta}}^{\alpha} \frac{\partial}{\partial z^{\alpha}} d z^{\bar{\beta}}
$$

associated to a complex tangent vector $\partial / \partial s^{i}$, namely

$$
\frac{\partial \ell\left(\gamma_{s}\right)}{\partial s^{i}}=\frac{1}{2} \int_{\gamma_{s}} A_{i} .
$$

In [A-S] we announced that the second variation of the geodesic length function could be treated with our methods.

Our Main result is Theorem 5.4, which provides a formula for the second variation of the length of closed geodesics in a holomorphic family of Kähler-Einstein manifolds. All further results are corollaries drawn from this theorem.

When dealing with tensors of higher order like curvature, which involve second order derivatives of metric tensors, certain integral operators arise in a natural way. In the context of automorphic forms the operator

$$
(\square+1)^{-1},
$$

where $\square$ denotes the (complex) Laplacian, was extensively studied (cf. [E]), and Wolpert used it in [WO2]. Later it played a major role in Siu's study of Kähler-Einstein manifolds [SIU] and also in [SCH1, SCH].

Its counterpart for geodesic integration rather than integration over the whole manifold is the operator

$$
\left(-\frac{D^{2}}{d t^{2}}+c\right)^{-1}, \quad c=1,2,
$$

where $D / d t$ denotes covariant differentiation along a geodesic.

Specializing our Theorem 5.4 to the case of families of Riemann surfaces we obtain the following theorem:

Theorem 1.2. Let $f: \mathcal{X} \rightarrow S$ be a holomorphic family of hyperbolic Riemann surfaces together with a differentiable family of closed geodesics $\gamma_{s}$. Then

$$
\frac{\partial^{2} \log \ell\left(\gamma_{s}\right)}{\partial s^{i} \partial s^{\bar{\jmath}}}=\frac{1}{2 \ell\left(\gamma_{s}\right)} \int_{\gamma_{s}}\left((\square+1)^{-1}\left(A_{i} A_{\bar{\jmath}}\right)+\left(-\frac{D^{2}}{d t^{2}}+2\right)^{-1}\left(A_{i}\right) \cdot A_{\bar{\jmath}}\right) .
$$

In the meantime our Theorem 1.1 was proved again in the case of Riemann surfaces in [W]. Also in [W] there is a computation of the second variation of the geodesic length function on Teichmüller space.

Let $P_{1}$ stand for a certain positive function depending on the diameter (precisely, a lower bound for the resolvent kernel), and recall that a finite set of closed geodesics 
in a hyperbolic Riemann surface $X$ with no boundary is said to fill up the surface if each component of the complement of their union is a cell.

Corollary 1.3. The following estimate holds for the second variation:

$$
\frac{\partial^{2} \log \ell\left(\gamma_{s}\right)}{\partial s^{i} \partial s^{\bar{\jmath}}} \geq \frac{1}{2} P_{1}\left(d\left(\mathcal{X}_{s}\right)\right) \cdot \int_{\mathcal{X}_{s}} A_{i} A_{\bar{\jmath}} g d V+\frac{1}{4 \ell\left(\gamma_{s}\right)^{2}} \int_{\gamma_{s}} A_{i} \int_{\gamma_{s}} A_{\bar{\jmath}} .
$$

In particular, $\log \ell\left(\gamma_{s}\right)$ is strictly plurisubharmonic.

Assume that the closed geodesics $\eta_{\nu}$ fill up the given Riemann surface. Then

$$
\log \sum_{\nu} \ell_{\nu}
$$

is a strictly plurisubharmonic exhaustion function.

(Inequalities " $\geq$ " and ">" for matrices are used in the sense that the difference is positive semi-definite and positive definite resp.)

Observe that the integral

$$
\int_{\mathcal{X}_{s}} A_{i} A_{\bar{\jmath}} g d V
$$

is just the Weil-Petersson inner product of the tangent vectors $\partial / \partial s^{i}$ and $\partial / \partial s^{j}$.

In the view of Theorem 1.1 the above estimate implies the following fact.

\section{Corollary 1.4.}

$$
\frac{\partial^{2} \log \ell\left(\gamma_{s}\right)}{\partial s^{i} \partial s^{\bar{\jmath}}}-\frac{\partial \log \ell\left(\gamma_{s}\right)}{\partial s^{i}} \frac{\partial \log \ell\left(\gamma_{s}\right)}{\partial s^{\bar{\jmath}}}>0 .
$$

Let a nodal complex curve represent a point at the boundary of the moduli space. If the node is given locally by the equation $z \cdot w=0$, then the opening-up of a node is a plumbing construction with a holomorphic paramenter $s$ for a degenerating holomorphic family, given by the equation $z \cdot w=s$. The holomorphic parameter $s$ corresponds to a closed geodesic $\gamma$, whose length tends to zero, when approaching the node.

The following estimate is known (cf. [WO5]):

$$
\ell\left(\gamma_{s}\right) \simeq 1 / \log \left(1 /|s|^{2}\right)
$$

If we insert the right-hand side into the expression (2) instead of $\ell\left(\gamma_{s}\right)$, we obtain the value zero. The estimate (2) implies:

Corollary 1.5. Let

$$
\ell\left(\gamma_{s}\right)=1 / \log (1 / \psi(s))
$$

for some positive function $\psi$. Then $\log \psi$ is strictly plurisubharmonic.

We consider the CR-submanifold given by the equation $\ell_{\gamma_{s}}=$ const. According to our theorem on the first variation of the geodesic length function, the complex tangent spaces $L$ with tangent vectors corresponding to harmonic Beltrami differentials $A$ are given by the equation

$$
\int_{\gamma_{s}} A=0 .
$$

This fact implies the following Corollary: 
Corollary 1.6. The Levi form on the complex tangent spaces $L$ of the CRsubmanifold given by the equation $\ell_{\gamma_{s}}=$ const. satisfies

$$
\left.\frac{\partial^{2} \log \ell\left(\gamma_{s}\right)}{\partial s^{i} \partial s^{\bar{\jmath}}}\right|_{L} \geq \frac{1}{2 \ell\left(\gamma_{s}\right)} \int_{\gamma_{s}}(\square+1)^{-1}\left(A_{i} A_{\bar{\jmath}}\right) .
$$

We have upper estimates:

Corollary 1.7. Let $\operatorname{dim} S=1$. Denote by $\left\|A_{s}\right\|_{0}$ the maximum of the pointwise norm of the harmonic Beltrami differential taken over the fiber $\mathcal{X}_{s}$. Then

$$
\frac{\partial^{2} \ell\left(\gamma_{s}\right)}{\partial s \partial \bar{s}} \leq \ell\left(\gamma_{s}\right)\left\|A_{s}\right\|_{0}^{2} \quad \text { and } \quad \frac{\partial^{2} \log \ell\left(\gamma_{s}\right)}{\partial s \partial \bar{s}} \leq \frac{3}{4}\left\|A_{s}\right\|_{0}^{2}
$$

Further applications are given in Section 6.

\section{Families of Kähler-Einstein Manifolds}

We compute the second variations of the geodesic length function in the general setting of Kähler-Einstein manifolds of negative Ricci curvature.

A Kähler form on a complex manifold $X$ of dimension $n$ will be denoted by

$$
\omega_{X}=\sqrt{-1} g_{\alpha \bar{\beta}} d z^{\alpha} \wedge d z^{\bar{\beta}} .
$$

We use the summation convention together with the $\nabla$-notation for covariant derivatives. A $\mid$-symbol will denote an ordinary derivative. Also, $\partial_{\alpha}$ and $\partial_{\bar{\beta}}$ will stand for $\partial / \partial z^{\alpha}$ and $\partial / \partial z^{\bar{\beta}}$ respectively. The raising and lowering of indices is defined as usual. We also use the semi-colon notation for covariant derivatives. For the Ricci tensor $R_{\alpha \bar{\beta}}$ on $X$ we use the sign convention

$$
R_{\alpha \bar{\beta}}=-\log (g(z))_{\mid \alpha \bar{\beta}},
$$

where $g(z)=\operatorname{det}\left(g_{\alpha \bar{\beta}}(z)\right)$. Furthermore $\left(g^{\bar{\beta} \alpha}\right)=\left(g_{\alpha \bar{\beta}}\right)^{-1}$.

Let $\left\{\mathcal{X}_{s}\right\}_{s \in S}$ be a holomorphic family of canonically polarized compact complex manifolds parameterized by a (connected) complex space $S$. It is given by a proper, smooth, holomorphic mapping $f: \mathcal{X} \rightarrow S$ such that $\mathcal{X}_{s}=f^{-1}(s)$ for all $s \in S$. For simplicity we will assume that the base $S$ is smooth, although our results can also be given a meaning for possibly non-reduced singular base spaces.

Local coordinates on $S$ will be denoted by $s^{i}, i=1, \ldots, N$. We use these as local coordinates on the total space $\mathcal{X}$ together with further local coordinates $z^{\alpha}$, $\alpha=1, \ldots, n$, where $n$ is the fiber dimension, satisfying $f(z, s)=s$.

The fibers $\mathcal{X}_{s}$ are equipped with Kähler-Einstein forms

$$
\omega_{\mathcal{X}_{s}}=\sqrt{-1} g_{\alpha \bar{\beta}}(z, s) d z^{\alpha} \wedge d z^{\bar{\beta}}
$$

depending smoothly upon the parameter $s$ and having constant negative Ricci curvature -1 . We write $g(z, s)=\operatorname{det}\left(g_{\alpha \bar{\beta}}(z, s)\right)$ and have $R_{\alpha \bar{\beta}}(z, s)=-g_{\alpha \bar{\beta}}(z, s)$.

We consider the real $(1,1)$-form

$$
\omega_{\mathcal{X}}=\sqrt{-1} \partial \bar{\partial} \log g(z, s)
$$

on the total space $\mathcal{X}$. The fiberwise Kähler-Einstein equation (3) implies that

$$
\omega_{\mathcal{X}} \mid \mathcal{X}_{s}=\omega_{\mathcal{X}}
$$

for all $s \in S$. In particular $\omega_{\mathcal{X}}$, restricted to any fiber, is positive definite. We will use the above notations introduced for the absolute case of a manifold $X$ concerning 
covariant derivatives, raising and lowering of indices, inverse of the metric tensor etc. also for the fibers in a holomorphic family.

The following fact is known:

Theorem. [SCH] Let $f: \mathcal{X} \rightarrow S$ be nowhere infinitesimally trivial. Then $\omega_{\mathcal{X}}$ is a Kähler form on the total space.

Let

$$
\rho: T_{s} S \rightarrow H^{1}\left(\mathcal{X}_{s}, \mathcal{T}_{\mathcal{X}_{s}}\right)
$$

be the Kodaira-Spencer map for the deformation $f: \mathcal{X} \rightarrow S$ at a point $s \in S$.

The Kähler-Einstein metric $\omega_{\mathcal{X}}$ on $\mathcal{X}_{s}$ induces a natural inner product on the space $H^{1}\left(\mathcal{X}_{s}, \mathcal{T}_{\mathcal{X}_{s}}\right)$ of infinitesimal deformations of $\mathcal{X}_{s}$ and thus on $T_{s} S$ via the map $\rho$; this is the Weil-Petersson Hermitian inner product on $T_{s} S$. Namely, given tangent vectors $u, v \in T_{s} S$, we denote by $A_{u}=A_{u \bar{\beta}}^{\alpha} \partial_{\alpha} d z^{\bar{\beta}}$ and $A_{v}$ the harmonic representatives of $\rho(u)$ and $\rho(v)$ respectively. Then the inner product of $u$ and $v$ equals

$$
\langle u, v\rangle_{W P}=\int_{\mathcal{X}_{s}} A_{u \bar{\beta}}^{\alpha} A_{\bar{v} \gamma}^{\bar{\delta}} g_{\alpha \bar{\delta}} g^{\bar{\beta} \gamma} g d V
$$

where $A_{\bar{v}}$ denotes the adjoint (conjugate) tensor of $A_{v}$, and $g d V$ the volume element.

We note that the Weil-Petersson inner product is positive definite at a given point of the base, if the induced deformation is effective.

We set $A_{j}=A_{\partial / \partial s^{j}}$. Then the Weil-Petersson form on $S$ equals

$$
\omega^{W P}=\sqrt{-1} G_{i \bar{\jmath}}^{W P}(s) d s^{i} \wedge d s^{\bar{\jmath}},
$$

where we use the notation

$$
G_{i \bar{\jmath}}^{W P}(s)=\left\langle\partial / \partial s^{i}, \partial / \partial s^{j}\right\rangle_{W P}=\int_{\mathcal{X}_{s}} A_{i \bar{\beta}}^{\alpha} A_{\bar{\jmath} \gamma}^{\bar{\delta}} g_{\alpha \bar{\delta}} g^{\bar{\beta} \gamma} g d V .
$$

The short exact sequence

$$
0 \rightarrow \mathcal{T}_{\mathcal{X} / S} \rightarrow \mathcal{T}_{\mathcal{X}} \rightarrow f^{*} \mathcal{T}_{S} \rightarrow 0
$$

induces the Kodaira-Spencer map via the edge homomorphism for direct images. A lift of a tangent vector $\partial / \partial s^{i}$ at a point $s$ of $S$ is a differentiable vector field on $\mathcal{X}_{s}$ with values in $\mathcal{T}_{\mathcal{X}}$. It has the form

$$
\partial / \partial s^{i}+b_{i}^{\alpha} \partial_{\alpha}
$$

Its exterior $\bar{\partial}$-derivative $B_{i \bar{\beta}}^{\alpha} \partial_{\alpha} d z^{\bar{\beta}}$, where $B_{i \bar{\beta}}^{\alpha}=\nabla_{\bar{\beta}} b_{i}^{\alpha}$, is interpreted as a $\bar{\partial}$-closed $(0,1)$-form on $\mathcal{X}_{s}$ with values in the tangent bundle of $\mathcal{X}_{s}$. Its cohomology class

$$
\rho\left(\partial / \partial s^{i}\right)=\left[B_{i \bar{\beta}}^{\alpha} \partial_{\alpha} d z^{\bar{\beta}}\right] \in H^{1}\left(\mathcal{X}_{s}, \mathcal{T}_{\mathcal{X}_{s}}\right) .
$$

equals the obstruction against the existence of a holomorphic lift of the given tangent vector, i.e. the infinitesimal triviality of the deformation in the direction of the tangent vector.

We now introduce notations that will be used in the rest of the paper.

The horizontal lift of $\partial / \partial s^{i}$, i.e. the lift that is perpendicular to the fibers with respect to $\omega_{\mathcal{X}}$ from (4), will be denoted by

$$
v_{i}=\partial / \partial s^{i}+a_{i}^{\alpha} \partial_{\alpha}
$$


Note that the quantities $a_{i}^{\alpha}$ are in general not tensors. It follows from the definition that

$$
a_{i}^{\alpha}=-g^{\bar{\beta} \alpha} g_{i \bar{\beta}}
$$

We set

$$
A_{i \bar{\beta}}^{\alpha}=\nabla_{\bar{\beta}} a_{i}^{\alpha} .
$$

The following properties of the tensors $A_{i \bar{\beta}}^{\alpha}$ are known (cf. [SCH1]) and will be used in the sequel:

Proposition 2.1. The horizontal lifts of tangent vectors with respect to $\omega_{\mathcal{X}}$ induce the harmonic representatives of Kodaira-Spencer classes in the sense that $A_{i \bar{\beta}}^{\alpha} \partial_{\alpha} d z^{\bar{\beta}}$ is the harmonic representative of $\rho\left(\partial / \partial s^{i}\right)$. The coefficients satisfy the following properties

$$
\begin{aligned}
\nabla_{\bar{\delta}} A_{i \bar{\beta}}^{\alpha} & =\nabla_{\bar{\beta}} A_{i \bar{\delta}}^{\alpha}, \\
\nabla_{\gamma} A_{i \bar{\beta}}^{\alpha} g^{\bar{\beta} \gamma} & =0 \\
A_{i \overline{\beta \delta}} & =A_{i \bar{\delta} \bar{\beta}} .
\end{aligned}
$$

The conditions (9) and (10) above correspond to harmonicity, whereas condition (11) reflects the relationship with the metric tensor.

We use the notation $c^{\bar{\beta}}=\overline{c^{\beta}}$ for (locally defined) tensors.

Later we will need the following fact:

Lemma 2.2. The partial derivatives of the Christoffel symbols with respect to the base parameter satisfy the identities

$$
\begin{aligned}
& \Gamma_{\gamma \sigma \mid s^{i}}^{\alpha}=-a_{i ; \gamma \sigma}^{\alpha}, \\
& \Gamma_{\gamma \sigma \mid s^{\bar{j}}}^{\alpha}=-g^{\bar{\beta} \alpha} a_{\bar{\jmath} \gamma ; \bar{\beta} \sigma} .
\end{aligned}
$$

\section{Families of closed geodesics}

Let $\left(f: \mathcal{X} \rightarrow S, \omega_{\mathcal{X}}\right)$ be a family of Kähler-Einstein manifolds with constant negative Ricci curvature -1 , where $\omega_{\mathcal{X}}$ is given by (4).

We denote by $\gamma_{s}$ a differentiable family of closed geodesics in the fibers $\mathcal{X}_{s}$, and by $\ell(s)$ the length of $\gamma_{s}$. In order to compute first and second variations, it is sufficient to assume that $S$ is a disk in the complex plane centered at 0 with coordinate $s$ (it is even sufficient to assume that the embedding dimension equals one). The general formulas follow from this case by polarization.

In local coordinates $(z, s)$ the closed geodesic curves $\gamma_{s}$ are solutions of the differential equation

$$
\ddot{u}^{\alpha}(t, s)+\Gamma_{\gamma \sigma}^{\alpha}(u(t, s)) \dot{u}^{\gamma}(t, s) \dot{u}^{\sigma}(t, s)=0 .
$$

The solution is unique up to an affine change of the parameter. In particular we may prescribe any positive constant value of its speed

$$
\|\dot{u}(t, s)\|^{2}=g_{\alpha \bar{\beta}}(u(t, s), s) \dot{u}^{\alpha}(t, s) \dot{u}^{\bar{\beta}}(t, s) .
$$

For $s=0$ we choose $\|\dot{u}\|=1$, for the remaining values of $s$ the value of $\|\dot{u}\|$ will be determined by the fact that the parameter $t$ assumes values in the interval $\left[0, \ell_{0}\right]$, 
where $\ell_{0}$ is the length of $\gamma_{0}$. Hence the family of geodesics is given by a map

$$
u: S \times\left[0, \ell_{0}\right] \rightarrow \mathcal{X}
$$

such that $f \circ u$ is the projection onto the first factor. Now

$$
u_{*}\left(\partial_{s}\right)=\partial_{s}+u_{s}^{\alpha} \partial_{\alpha}+u_{s}^{\bar{\beta}} \partial_{\bar{\beta}}
$$

with partial derivatives

$$
u_{s}^{\alpha}:=u_{\mid s}^{\alpha} \text { and } u_{s}^{\bar{\beta}}:=\left(\overline{u^{\beta}}\right)_{\mid s} .
$$

Note that the $(1,0)$ - and $(0,1)$-components $\partial_{s}+u_{s}^{\alpha} \partial_{\alpha}$ and $u_{s}^{\bar{\beta}} \partial_{\bar{\beta}}$ of $u_{*} \partial_{s}$ are tensors along the geodesics with values in $T_{\mathcal{X}}$ and $T_{\mathcal{X} / S} \subset T_{\mathcal{X}}$ respectively. In a similar way the tensor

$$
\dot{u}=u_{*}(d / d t)=\dot{u}^{\alpha} \partial_{\alpha}+\dot{u}^{\bar{\beta}} \partial_{\bar{\beta}}
$$

along the family of geodesics has a type decomposition. The difference of two lifts of tangent vectors from the base is a tangent vector along the geodesics (with values in the relative tangent bundle). For $\operatorname{dim} S=1$ we have the horizontal lift

$$
v_{s}=\partial_{s}+a^{\alpha} \partial_{\alpha} .
$$

The difference of $u_{*}\left(\partial_{s}\right)$ and the horizontal lift has the components

$$
\begin{aligned}
u_{s}^{\alpha}-a_{s}^{\alpha} & =u_{s}^{\alpha}(s, t)-a_{s}^{\alpha}(u(s, t), s), \\
u_{s}^{\bar{\beta}} & =u_{s}^{\bar{\beta}}(s, t) .
\end{aligned}
$$

For any tensor along the geodesic $\gamma_{s}$ on a fiber $\mathcal{X}_{s}$ we denote by $D / d t$ the covariant derivative along $\gamma_{s}$. In particular

$$
\frac{D}{d t} \dot{u}=0
$$

Let $w^{\alpha}(t) \partial_{\alpha}$ be any vector field along $\gamma_{s}$. Then

$$
\frac{D}{d t} w^{\alpha}(t)=\dot{w}^{a}(t)+\Gamma_{\gamma \sigma}^{\alpha}(u(t)) w^{\gamma}(t) \dot{u}^{\sigma}(t) .
$$

If $w^{\alpha}(t)$ is of the form $\widetilde{w}^{\alpha}(u(t))$, then (21) implies

$$
\frac{D}{d t} w^{\alpha}(t)=\widetilde{w}^{\alpha}(u(t))_{; \kappa} \dot{u}^{\kappa}(t)+\widetilde{w}^{\alpha}(u(t))_{; \bar{\lambda}} \dot{u}^{\bar{\lambda}}(t) .
$$

Corresponding equations hold for tensors of type $(0,1)$ and contravariant tensors.

Lemma 3.1. We have

$$
\begin{aligned}
\frac{D}{d t}\left(u_{s}^{\alpha}-a_{s}^{\alpha}\right) & =\dot{u}_{s}^{\alpha}+\Gamma_{\gamma \sigma}^{\alpha} u_{s}^{\gamma} \dot{u}^{\sigma}-a_{s ; \gamma}^{\alpha} \dot{u}^{\gamma}-A_{s \bar{\beta}}^{\alpha} \dot{u}^{\bar{\beta}} \\
\frac{D^{2}}{d t^{2}}\left(u_{s}^{\alpha}-a_{s}^{\alpha}\right) & =R_{\sigma \gamma \bar{\lambda}}^{\alpha} \dot{u}^{\sigma} \dot{u}^{\bar{\lambda}}\left(u_{s}^{\gamma}-a_{s}^{\gamma}\right)-R_{\sigma \gamma \bar{\lambda}}^{\alpha} u_{s}^{\bar{\lambda}} \dot{u}^{\gamma} \dot{u}^{\sigma}-2 A_{s \bar{\delta} ; \gamma}^{\alpha} \dot{u}^{\gamma} \dot{u}^{\bar{\delta}}-A_{s \bar{\delta} ; \bar{\tau}}^{\alpha} \dot{u}^{\bar{\delta}} \dot{u}^{\bar{\tau}} \\
\frac{D}{d t}\left(u_{s}^{\bar{\beta}}\right) & =\dot{u}_{s}^{\bar{\beta}}+\Gamma_{\bar{\delta} \bar{\tau}}^{\bar{\beta}} u_{s}^{\bar{\delta}} \dot{u}^{\bar{\tau}} \\
\frac{D^{2}}{d t^{2}}\left(u_{s}^{\bar{\beta}}\right) & =g^{\bar{\beta} \alpha} A_{s \bar{\delta} \bar{\tau} ; \alpha} \dot{u}^{\bar{\delta}} \dot{u}^{\bar{\tau}}-R_{\bar{\delta} \bar{\tau} \sigma}^{\bar{\beta}}\left(u_{s}^{\sigma}-a_{s}^{\sigma}\right) \dot{u}^{\bar{\tau}} \dot{u}^{\bar{\delta}}+R_{\bar{\delta} \bar{\tau} \sigma}^{\bar{\beta}} \dot{u}^{\sigma} \dot{u}^{\bar{\tau}} u_{s}^{\bar{\delta}}
\end{aligned}
$$


Proof. The equations (23) and (25) follow immediately from the definition. The remaining proofs are rather computational: To prove (24) we apply $D / d t$ to $(23)$ and differentiate (14) with respect to $s$. In this way we can eliminate $\ddot{u}_{s}^{\alpha}$. We use (12), and finally we have (24). Observe that we need to consider both ordinary and covariant derivatives of Christoffel symbols. We prove (26) in the same way.

In order to describe the variation of the length of closed geodesics in a family, we use the notion of integrating a tensor along a geodesic. Exemplarily we define:

Definition 3.2. Let $C=C_{\overline{\beta \delta}}$ be a tensor on the Kähler manifold $X$, and $\gamma$ be a geodesic of length $\ell$, parameterized by $u(t)=\left(u^{1}(t), \ldots, u^{n}(t)\right)$, such that $\|\dot{u}(t)\|=1$. Then

$$
\int_{\gamma} C=\int_{\gamma} C_{\overline{\beta \delta}} d z^{\bar{\beta}} d z^{\bar{\delta}}:=\int_{0}^{\ell} C_{\overline{\beta \delta}}(u(t)) \dot{u}^{\bar{\beta}} \dot{u}^{\bar{\delta}} d t .
$$

For contravariant tensors of order one this notation coincides with the integration of a differential form along the curve $\gamma$. For covariant tensors the geodesic integral is defined after lowering indices with respect to the metric tensor.

\section{First variation of the geodesic length function}

Given a holomorphic family of Kähler-Einstein manifolds with one dimensional base space like in the previous section together with a differentiable family of closed geodesics $\gamma_{s}$ with parametrization (15), the length of these is equal to

$$
\ell(s)=\int_{0}^{\ell_{0}}\|\dot{u}(t, s)\| d t
$$

so that

$$
\left.\frac{d \ell(s)}{d s}\right|_{s=0}=\frac{1}{2} \int_{0}^{\ell_{0}} \frac{d}{d s}\|\dot{u}(t, s)\|^{2} d t
$$

We will compute

$$
\frac{d}{d s}\|\dot{u}(t, s)\|^{2}=\frac{d}{d s}\left(g_{\alpha \bar{\beta}} \dot{u}^{\alpha} \dot{u}^{\bar{\beta}}\right) .
$$

We denote by $\langle,\rangle_{\mathcal{X}}$ the inner product with respect to $\omega_{\mathcal{X}}$.

Lemma 4.1. We have

$$
\frac{d}{d s}\left(g_{\alpha \bar{\beta}} \dot{u}^{\alpha} \dot{u}^{\bar{\beta}}\right)-\frac{d}{d t}\left\langle u_{*} \partial_{s}, \dot{u}\right\rangle_{\omega_{\mathcal{X}}}=A_{s \overline{\beta \delta}} \dot{u}^{\bar{\beta}} \dot{u}^{\bar{\delta}} .
$$

In the computational proof one uses (20), (12), (23), and (25).

An immediate consequence of the above Lemma is Theorem 1.1.

\section{Second variation of the geodesic length function}

An important function is given by the inner product of harmonic lifts of tangent vectors. In terms of local holomorphic coordinates $s^{i}$ on $S$ (or coordinates of a smooth ambient space of minimal dimension at a given point of the base) we have:

Definition 5.1. Let $v_{i}$ be the horizontal lift of $\partial / \partial s^{i}$. We put

$$
\varphi_{i \bar{\jmath}}=\left\langle v_{i}, v_{j}\right\rangle_{\mathcal{X}}
$$

where the inner product is taken pointwise. 
We list basic properties of the function $\varphi_{i \bar{j}}$ on $\mathcal{X}$ :

$$
\begin{aligned}
\varphi_{i \bar{\jmath}} & =g_{i \bar{\jmath}}-a_{i}^{\alpha} a_{\bar{\jmath}}^{\bar{\beta}} g_{\alpha \bar{\beta}}, \\
(\square+1) \varphi_{i \bar{\jmath}} & =\left\langle A_{i \overline{\beta \delta}}, A_{j \bar{\lambda} \bar{\tau}}\right\rangle=A_{i \bar{\beta}}^{\alpha} A_{\bar{\jmath} \alpha}^{\bar{\beta}}, \\
\int_{\mathcal{X}_{s}} \varphi_{i \bar{\jmath}} & =G_{i \bar{\jmath}}^{W P}, \\
\omega_{\mathcal{X}}^{n+1} & =\sqrt{-1} \varphi_{i \bar{\jmath}} d s^{i} \wedge d s^{\bar{\jmath}} \wedge \omega_{\mathcal{X}}^{n} .
\end{aligned}
$$

The first of these equalities follows from the definition. For the second equality cf. [SCH, Proposition 2]. The equation (32) follows from (31). The last equation (33) is Lemma 6 from $[\mathrm{SCH}]$.

We will apply the following fact:

Theorem. $[\mathrm{SCH}]$ The relative canonical bundle $\mathcal{K}_{\mathcal{X} / S}$ equipped with the hermitian metric induced by the relative Kähler-Einstein forms is positive, i.e. the matrix $\left(\varphi_{i \bar{\jmath}}\right)$ is positive definite.

The lower estimates for $\left(\varphi_{i \bar{\jmath}}\right)$ from [SCH] will be applied below.

Again, it is sufficient to do computations for a base space $S$ of dimension one with coordinate $s$. By abuse of notation, we use $s$ and $\bar{s}$ as indices instead of $i$ and $\bar{\jmath}$, where $i, j$ can only take the value 1 .

Lemma 5.2. We have

$$
A_{s \overline{\beta \delta} \mid \bar{s}}=-\varphi_{s \bar{s} ; \overline{\beta \delta}}-A_{s \bar{\tau} \bar{\beta} ; \bar{\delta}} a_{\bar{s}}^{\bar{\tau}}-A_{s \bar{\tau} \bar{\beta}} a_{\bar{s} ; \bar{\delta}}^{\bar{\tau}}-A_{s \bar{\tau} \bar{\delta}} a_{\bar{s} ; \bar{\beta}}^{\bar{\tau}} .
$$

Proof. We compute

$$
A_{s \overline{\beta \delta} \mid \bar{s}}=\left(a_{s \bar{\beta} \mid \bar{\delta}}+a_{s \bar{\tau}} \Gamma_{\bar{\beta} \delta}^{\bar{\tau}}\right)_{\bar{s}}=a_{s \bar{\beta} \mid \bar{s} ; \bar{\delta}}+a_{s \bar{\tau}} \Gamma_{\bar{\beta} \delta \mid \bar{s}}^{\bar{\tau}} .
$$

Now the claim follows from (12) and (30).

From here we immediately obtain the following identity.

Lemma 5.3. We have

$$
\begin{aligned}
\frac{\partial}{\partial \bar{s}}\left(A_{s \overline{\beta \delta}} \dot{u}^{\bar{\beta}} \dot{u}^{\bar{\delta}}\right)= & \left(-\varphi_{s \bar{s} ; \overline{\beta \delta}}-A_{s \bar{\tau} \bar{\beta} ; \bar{\delta}} a_{\bar{s}}^{\bar{\tau}}-2 A_{s \bar{\tau} \bar{\beta}} a_{\bar{s} ; \bar{\delta}}^{\bar{\tau}}\right) \dot{u}^{\bar{\beta}} \dot{u}^{\bar{\delta}} \\
& +A_{s \overline{\beta \delta} \mid \bar{\tau}} u_{\bar{s}}^{\bar{\tau}} \dot{u}^{\bar{\beta}} \dot{u}^{\bar{\delta}}+A_{s \overline{\beta \delta} ; \gamma} u_{\bar{s}}^{\gamma} \dot{u}^{\bar{\beta}} \dot{u}^{\bar{\delta}}+2 A_{s \overline{\beta \delta}} \dot{u}_{\bar{s}}^{\bar{\beta}} \dot{u}^{\bar{\delta}} .
\end{aligned}
$$

We need to eliminate mixed derivatives in the parameters $t$ and $s$. We define a function $\chi$ along the geodesics by the formula

$$
\chi=\left\langle A_{s \bar{\lambda}}^{\kappa} \dot{u}^{\bar{\lambda}} \partial_{\kappa}, u_{*}\left(\partial_{s}\right)\right\rangle_{\omega_{\mathcal{X}}}=A_{s \overline{\beta \delta}}\left(u_{\bar{s}}^{\bar{\beta}}-a_{\bar{s}}^{\bar{\beta}}\right) \dot{u}^{\bar{\delta}}
$$

and obtain

$$
\frac{d}{d t} \chi=\frac{D}{d t}\left(A_{s \overline{\beta \delta}}\right)\left(u_{\bar{s}}^{\bar{\beta}}-a_{\bar{s}}^{\bar{\beta}}\right) \dot{u}^{\bar{\delta}}+A_{s \overline{\beta \delta}} \frac{D}{d t}\left(u_{\bar{s}}^{\bar{\beta}}-a_{\bar{s}}^{\bar{\beta}}\right) \dot{u}^{\bar{\delta}} .
$$

A straightforward calculation using the identities (22) and (23) shows that

$$
\begin{aligned}
\frac{\partial}{\partial \bar{s}}\left(A_{s \overline{\beta \delta}} \dot{u}^{\bar{\beta}} \dot{u}^{\bar{\delta}}\right)-2 \dot{\chi}+\frac{d}{d t}\left(\varphi_{s \bar{s} ; \bar{\beta}} \dot{u}^{\bar{\beta}}\right)= & \left(\varphi_{s \bar{s} ; \alpha \bar{\beta}}+2 A_{s \overline{\beta \delta}} A_{\bar{s} \alpha}^{\bar{\delta}}\right) \dot{u}^{\alpha} \dot{u}^{\bar{\beta}} \\
& -\left(A_{s \overline{\beta \delta} ; \bar{\tau}} \dot{u}^{\bar{\tau}}+A_{s \overline{\beta \delta} ; \gamma} \dot{u}^{\gamma}\right)\left(u_{\bar{s}}^{\bar{\beta}}-a_{\bar{s}}^{\bar{\beta}}\right) \dot{u}^{\bar{\delta}} \\
& +A_{s \overline{\beta \delta} ; \gamma} \dot{u}^{\bar{\delta}}\left(u_{\bar{s}}^{\gamma} \dot{u}^{\bar{\beta}}-\left(u_{\bar{s}}^{\bar{\beta}}-a_{\bar{s}}^{\bar{\beta}}\right) \dot{u}^{\gamma}\right) .
\end{aligned}
$$


This concludes the first part of the computation. Altogether we obtained:

Theorem 5.4. Let $\mathcal{X} \rightarrow S$ be a holomorphic family of Kähler-Einstein manifolds of constant negative Ricci curvature together with a differentiable family of closed geodesics. Then the second variation of the geodesic length function equals

$$
\begin{gathered}
\frac{\partial^{2} \ell(s)}{\partial s \partial \bar{s}}=\frac{1}{2} \int_{\gamma_{s}}\left(\left(\varphi_{s \bar{s} ; \alpha \bar{\beta}}+2 A_{s \overline{\beta \delta}} A_{\bar{s} \alpha}^{\bar{\delta}}\right) \dot{u}^{\alpha} \dot{u}^{\bar{\beta}}-\left(A_{s \overline{\beta \delta} ; \bar{\tau}} \dot{u}^{\bar{\tau}}+A_{s \overline{\beta \delta} ; \gamma} \dot{u}^{\gamma}\right)\left(u_{\bar{s}}^{\bar{\beta}}-a_{\bar{s}}^{\bar{\beta}}\right) \dot{u}^{\bar{\delta}}\right. \\
\left.+A_{s \overline{\beta \delta} ; \gamma} \dot{u}^{\bar{\delta}}\left(u_{\bar{s}}^{\gamma} \dot{u}^{\bar{\beta}}-\left(u_{\bar{s}}^{\bar{\beta}}-a_{\bar{s}}^{\bar{\beta}}\right) \dot{u}^{\gamma}\right)\right) .
\end{gathered}
$$

An intrinsic version will be given in Section 10.

\section{Second variation of the geodesic length function on Teichmüller spaces}

From now on we assume that fibers of $f: \mathcal{X} \rightarrow S$ are one dimensional. We set $z=z^{1}$ and also use $z$ and $\bar{z}$ as indices. The preceding formulas and the notation remain valid, if the fibers are equipped with the hyperbolic metric of constant Ricci curvature -1 , i.e. on a fiber $\mathcal{X}_{s}$ with coordinate function $z$ we have

$$
d s^{2}=g(z, s) \sqrt{-1} d z \wedge \overline{d z}
$$

where $g(z, s)$ satisfies the equation

$$
g(z, s)=\frac{\partial^{2} \log g(z, s)}{\partial z \overline{\partial z}} .
$$

Free homotopy classes of simple closed curves are represented by closed geodesics $\gamma_{s}$ with parameterization $u(s, t)$, which depend in a differentiable way upon the parameter $s$.

According to our general index convention we have $g=g_{z \bar{z}}$. Observe that the harmonic Kodaira-Spencer form

$$
A_{s \bar{z}}^{z}(z) \partial_{z} \overline{d z}=: \mu(z) \partial_{z} \overline{d z}
$$

is exactly a harmonic Beltrami differential. Likewise

$$
A_{\bar{s} z z}(z)=g_{z \bar{z}}(z) A_{\bar{s} z}^{\bar{z}}(z)=g_{z \bar{z}} \bar{\mu}(z)
$$

defines a holomorphic quadratic differential. The statement of Theorem 5.4 now reads as follows:

Proposition 6.1. We have

$$
\frac{\partial^{2} \ell\left(\gamma_{s}\right)}{\partial s \partial \bar{s}}=\frac{1}{2} \int_{\gamma_{s}}\left(\left(\varphi_{s \bar{s}}+g^{\bar{z} z} A_{s \bar{z} \bar{z}} A_{\bar{s} z}^{\bar{z}}\right)-\frac{D}{d t} A_{s \bar{z} \bar{z}} \dot{u}^{\bar{z}}\left(u_{\bar{s}}^{\bar{z}}-a_{\bar{s}}^{\bar{z}}\right)\right) .
$$

Proof. In dimension one, the term that involves the function $\varphi$ can be interpreted as a complex Laplacian and (31) is applicable. We use gi $\dot{\bar{u}}=1$. The harmonicity of the Kodaira-Spencer tensor is equivalent to

$$
A_{s \bar{z} \bar{z} ; z}=0
$$

so that the latter terms in (36) vanish. 
Theorem 6.2. Let $f: \mathcal{X} \rightarrow S$ be a holomorphic family of hyperbolic Riemann surfaces together with a differentiable family of closed geodesics $\gamma_{s}$. Then

$$
\begin{aligned}
\frac{\partial^{2} \ell\left(\gamma_{s}\right)}{\partial s^{i} \partial s^{\bar{\jmath}}}= & \frac{1}{2} \int_{\gamma_{s}}\left((\square+1)^{-1}\left(A_{i} \cdot A_{\bar{\jmath}}\right)+\left(-\frac{D^{2}}{d t^{2}}+2\right)^{-1}\left(A_{i}\right) \cdot A_{\bar{\jmath}}\right) \\
& +\frac{1}{4 \ell\left(\gamma_{s}\right)} \int_{\gamma_{s}} A_{i} \cdot \int_{\gamma_{s}} A_{\bar{\jmath}} .
\end{aligned}
$$

This statement, together with Theorem 1.1 implies our Theorem 1.2. The computation [W, Theorem 1.1] seems to be related.

We prove the above theorem in Section 8.

We estimate the integrand in (38) from below:

Definition 6.3. Given any two Hermitian symmetric matrices $M_{i \bar{\jmath}}$ and $N_{i \bar{\jmath}}$, we write $M_{i \bar{\jmath}} \geq N_{i \bar{\jmath}}$, if the difference is a positive semi-definite matrix.

Corollary 6.4. We have the inequality

$$
\frac{\partial^{2} \ell\left(\gamma_{s}\right)}{\partial s^{i} \partial s^{\bar{\jmath}}} \geq \frac{1}{2}\left(\int_{\gamma_{s}}(\square+1)^{-1}\left(A_{i} \cdot A_{\bar{\jmath}}\right)+\frac{1}{\ell\left(\gamma_{s}\right)} \int_{\gamma_{s}} A_{i} \int_{\gamma_{s}} A_{\bar{\jmath}}\right)
$$

in the sense of Definition 6.3. In particular the geodesic length function is strictly plurisubharmonic.

Again we apply Theorem 1.1 and obtain the following statement.

Corollary 6.5. The logarithm of the geodesic length function is strictly plurisubharmonic: The inequality

$$
\begin{aligned}
\frac{\partial^{2} \log \ell\left(\gamma_{s}\right)}{\partial s^{i} \partial s^{\bar{\jmath}}} & \geq \frac{1}{2 \ell\left(\gamma_{s}\right)} \int_{\gamma_{s}}(\square+1)^{-1}\left(A_{i} \cdot A_{\bar{\jmath}}\right)+\frac{1}{4 \ell\left(\gamma_{s}\right)^{2}} \int_{\gamma_{s}} A_{i} \int_{\gamma_{s}} A_{\bar{\jmath}} \\
& =\frac{1}{2 \ell\left(\gamma_{s}\right)} \int_{\gamma_{s}}(\square+1)^{-1}\left(A_{i} \cdot A_{\bar{\jmath}}\right)+\frac{\partial \log \ell\left(\gamma_{s}\right)}{\partial s^{i}} \frac{\partial \log \ell\left(\gamma_{s}\right)}{\partial s^{\bar{\jmath}}}
\end{aligned}
$$

holds in the sense of Definition 6.3.

A lower estimate for the functions $\varphi_{i \bar{\jmath}}=(\square+1)^{-1}\left(A_{i} \cdot A_{\bar{\jmath}}\right)$ is known:

Proposition. (cf. [F]) There exists a positive function $P_{1}\left(d\left(\mathcal{X}_{s}\right)\right)$, which depends on the diameter of $\mathcal{X}_{s}$, such that for any solution

$$
(\square+1) \varphi=\chi,
$$

with $\chi \geq 0$ the inequality

$$
\varphi(z) \geq P_{1}\left(d\left(\mathcal{X}_{s}\right)\right) \int_{\mathcal{X}_{s}} \chi g d V
$$

holds for all $z \in \mathcal{X}_{s}$.

The above proposition implies the following estimate, which can be used together with Corollary 6.4 and Corollary 6.5 to obtain further inequalities like Corollary 1.3:

$$
\int_{\gamma_{s}}(\square+1)^{-1}\left(A_{i} \cdot A_{\bar{\jmath}}\right) \geq \ell\left(\gamma_{s}\right) \cdot P_{1}\left(d\left(\mathcal{X}_{s}\right)\right) \cdot G_{i \bar{\jmath}}^{W P} .
$$


Lemma 6.6. (cf. [SCH2, Lemma 3]) Let $\ell_{j}$ be positive functions on a complex manifold. Then the following estimate of closed hermitian $(1,1)$-forms holds:

$$
\sqrt{-1} \partial \bar{\partial} \log \left(\sum_{j} \ell_{j}\right) \geq \frac{1}{\sum_{k} \ell_{k}} \sum_{j}\left(\ell_{j} \sqrt{-1} \partial \bar{\partial} \log \ell_{j}\right) .
$$

The above Lemma implies that estimates for the single geodesic length functions carry over to any sum of such functions. Kerckhoff showed in $[\mathrm{K}]$ that for a finite number of closed geodesics $\gamma_{1}, \ldots, \gamma_{m}$, which fill up the Riemann surface the sum of the geodesic length functions provides a proper exhaustion of the Teichmüller space. Wolpert proved in [WO3] that the function $\left(\ell\left(\gamma_{1}\right)+\ldots+\ell\left(\gamma_{m}\right)\right)^{1 / 2}$ is actually convex along the Weil-Petersson geodesics and $\log \left(\ell\left(\gamma_{1}\right)+\ldots+\ell\left(\gamma_{m}\right)\right)$ is strictly plurisubharmonic (cf. [WO4, WO5]).

Yeung constructs in [Ye] a bounded non-positive strictly plurisubharmonic exhaustion function. His estimates of the second variation of the geodesic length function follow from ours.

Corollary 6.7. The logarithm of any sum of geodesic length functions is strictly plurisubharmonic with estimates given by Lemma 6.6.

We conclude the section with the proof of upper estimates which we state for $\operatorname{dim} S=1$.

Proof of Corollary 1.7. The maximum principle applied to the equation (31) yields that

$$
\varphi_{s \bar{s}}(z) \leq\left\|A_{s}\right\|_{0}^{2}
$$

Furthermore,

$$
\int_{\gamma_{s}}\left(2-D^{2} / d t^{2}\right)^{-1}\left(A_{s}\right) \cdot A_{\bar{s}} \leq \frac{1}{2} \int_{\gamma_{s}} A_{s} \cdot A_{\bar{s}} \leq \frac{1}{2} \ell\left(\gamma_{s}\right)\left\|A_{s}\right\|_{0}^{2},
$$

and finally

$$
\left|\int_{\gamma_{s}} A_{s}\right|^{2} \leq \ell\left(\gamma_{s}\right) \int_{\gamma_{s}} A_{s} \cdot A_{\bar{s}}
$$

These estimates imply both inequalities.

\section{Differential operators along closed geodesics}

When studying covariant differentiation along geodesics $u(t)$ on a fixed Riemann surface, we observe that the obvious identities

$$
\frac{D}{d t} \dot{u}=0 \quad \text { and } \quad \frac{D}{d t} g_{z \bar{z}}=0
$$

can be used to reduce covariant differentiation of tensors along a closed geodesic to the (covariant) differentiation of functions. In our case all functions will be of class $C^{\infty}$. Hilbert space theory and regularity theorems are available and need not explicitly be mentioned.

Lemma 7.1. The operator

$$
L=-\frac{D^{2}}{d t^{2}}+1
$$

is invertible with bounded inverse. 

by

Let $\lambda_{\nu} \geq 0, \lambda_{0}=0$ be the eigenvalues of $-D^{2} / d t^{2}$. For any function $\psi$ we denote

$$
\psi=\sum_{\nu \geq 0} \psi_{\nu}
$$

the eigenvector decomposition. An inverse of the operator $L-L^{-1}$ is defined on the orthogonal complement $C$ of the kernel of $D^{2} / d t^{2}$ (which is also the kernel of $D / d t$ ) with values in the same complement.

Lemma 7.2. Let

$$
M=\left(L-L^{-1}\right)^{-1} \circ\left(-\frac{D^{2}}{d t^{2}}\right)
$$

Then

$$
M(\psi)=\sum_{\nu>0}\left(1-\frac{1}{2+\lambda_{\nu}}\right) \psi_{\nu}
$$

In particular,

$$
M=1-\left(2-\frac{D^{2}}{d t^{2}}\right)^{-1}-\frac{1}{2} H
$$

where $H$ denotes the harmonic projection.

\section{Proof of Theorem 6.2}

We now prove Theorem 6.2. Only the case of $\operatorname{dim} S=1$ is needed.

For one dimensional fibers (24) and (26) read

$$
\begin{aligned}
\frac{D^{2}}{d t^{2}}\left(u_{s}^{z}-a_{s}^{z}\right) & =\left(u_{s}^{z}-a_{s}^{z}\right)-g_{z \bar{z}} \dot{u}^{z} \dot{u}^{z} u_{s}^{\bar{z}}-A_{s \bar{z} ; \bar{z}}^{z} \dot{u}^{\bar{z}} \dot{u}^{\bar{z}}, \\
\frac{D^{2}}{d t^{2}}\left(u_{\bar{s}}^{z}\right) & =-g_{z \bar{z}}\left(u_{\bar{s}}^{\bar{z}}-a_{\bar{s}}^{\bar{z}}\right) \dot{u}^{z} \dot{u}^{z}+u_{\bar{s}}^{z} .
\end{aligned}
$$

We define auxiliary functions along the geodesics. Let

$$
w=\left(u_{s}^{z}-a_{s}^{z}\right) \dot{u}^{\bar{z}} g_{z \bar{z}}, \quad v=u_{s}^{\bar{z}} \dot{u}^{z} g_{z \bar{z}}, \quad A=A_{s \bar{z} \bar{z}}\left(\dot{u}^{\bar{z}}\right)^{2} .
$$

We apply (39) and (40) and use the notation of the preceding paragraph. The aim is to express the function $w$ in terms of the Kodaira-Spencer form. We have

$$
L w=v+\frac{D}{d t} A, \quad L v=w, \quad \frac{D}{d t} A=\left(L-L^{-1}\right) w .
$$

The derivative $(D / d t)(A)$ is orthogonal to the kernel of $D^{2} / d t^{2}$, so that $\left(L-L^{-1}\right)^{-1}$ is defined in the sense of the previous section.

In this shorthand notation, Proposition 6.1 reads

$$
2 \frac{\partial^{2} \ell\left(\gamma_{s}\right)}{\partial s \partial \bar{s}}=\int_{\gamma_{s}}\left(\varphi+|A|^{2}-w \cdot \frac{D}{d t}(\bar{A})\right) .
$$

Modulo terms whose integral vanish

$$
\begin{aligned}
-\frac{D}{d t} A \cdot \bar{w} & =-\frac{D}{d t}(A) \cdot\left(L-L^{-1}\right)^{-1}\left(\frac{D}{d t}(\bar{A})\right) \equiv\left(L-L^{-1}\right)^{-1}\left(\frac{D^{2}}{d t^{2}}(A)\right) \cdot \bar{A} \\
& =-M(A) \cdot \bar{A}=-\left(1-\left(2-\frac{D^{2}}{d t^{2}}\right)^{-1}\right)(A) \cdot \bar{A}+\frac{1}{2} H(A) \cdot \bar{A} .
\end{aligned}
$$


Hence, writing $\ell$ for $\ell\left(\gamma_{s}\right)$, we have

$$
\begin{aligned}
\frac{\partial^{2} \ell}{\partial s \partial \bar{s}} & =\frac{1}{2} \int_{\gamma_{s}}\left(\varphi+\left(\frac{D^{2}}{d t^{2}}+2\right)^{-1}(A) \cdot \bar{A}\right)+\frac{1}{4 \ell}\left|\int_{\gamma_{s}} A\right|^{2} \\
& =\frac{1}{2} \int_{\gamma_{s}}\left((\square+1)^{-1}\left(|A|^{2}\right)+\left(-\frac{D^{2}}{d t^{2}}+2\right)^{-1}(A) \cdot \bar{A}\right)+\frac{1}{\ell}\left|\frac{\partial \ell}{\partial s}\right|^{2},
\end{aligned}
$$

and

$$
\frac{\partial^{2} \log \ell}{\partial s \partial \bar{s}}=\frac{1}{2 \ell} \int_{\gamma_{s}}\left((\square+1)^{-1}\left(|A|^{2}\right)+\left(-\frac{D^{2}}{d t^{2}}+2\right)^{-1}(A) \cdot \bar{A}\right) .
$$

\section{Weighted punctured Riemann surfaces and conical metrics}

Our methods equally apply to the case of punctured Riemann surfaces. In our previous paper [A-S], we discussed the first variation of the geodesic length function for Teichmüller spaces of weighted punctured Riemann surfaces equipped with hyperbolic conical metrics. Using the extended techniques in [S-T] one can see that our results on second variations and plurisubharmonicity hold true in the conical case (for weights $\geq 1 / 2$ ).

\section{Remarks on the higher dimensional case}

Rather little seems to be known about minimal closed geodesics on non-rigid canonically polarized varieties.

The aim is to express the second variation of the length of closed geodesics in a holomorphic family only in terms of the variation of the complex structure.

We will first give an intrinsic version of Theorem 5.4 and introduce the following notation in the situation of Section 1.1.

Let $x=x^{\alpha} \partial_{\alpha}, y=y^{\alpha} \partial_{\alpha}$ etc. be differentiable vector fields along the given geodesic. We set:

$$
\begin{aligned}
\partial A(\bar{x}, y) & =A_{s \bar{\beta} ; \gamma}^{\alpha} x^{\bar{\beta}} y^{\gamma} \partial_{\alpha}, \\
\partial A(\bar{x}, \bar{y}) & =A_{s \bar{\beta} ; \bar{\delta}}^{\alpha} x^{\bar{\beta}} y^{\bar{\delta}} \partial_{\alpha}, \\
\partial A(\bar{x}, \bar{y}, z) & =A_{s \overline{\beta \delta} ; \gamma} x^{\bar{\beta}} y^{\bar{\delta}} z^{\gamma}, \\
\bar{\partial} A(\bar{x}, \bar{y}, \bar{z}) & =A_{s \overline{\beta \delta} ; \bar{\tau}} x^{\bar{\beta}} y^{\bar{\delta}} z^{\bar{\tau}}, \\
(\uparrow \partial A)(\bar{x}, \bar{y}) & =A_{s \overline{\beta \delta}}^{; \gamma} x^{\bar{\beta}} y^{\bar{\delta}} \partial_{\gamma}, \\
R(x, \bar{y})(z) & =R_{\gamma \sigma \bar{\tau}}^{\alpha} x^{\sigma} y^{\bar{\tau}} z^{\gamma} \partial_{\alpha}, \\
R(\bar{x}, y)(\bar{z}) & =R_{\bar{\delta} \overline{\bar{\tau} \sigma}}^{\bar{\beta}} x^{\bar{\tau}} y^{\sigma} z^{\bar{\delta}} \partial_{\bar{\beta}} .
\end{aligned}
$$

We are already given the following vector fields along the closed geodesic:

$$
\dot{u}=\dot{u}^{\alpha} \partial_{\alpha}, \quad v=u_{\bar{s}}^{\alpha} \partial_{\alpha}, \quad w=\left(u_{s}^{\alpha}-a_{s}^{\alpha}\right) \partial_{\alpha} .
$$

We denote by Hess the complex Hessian. Now (36) can be expressed in the following way (with obvious abbreviations): 
Theorem 10.1. We have

$$
\begin{aligned}
\frac{\partial^{2} \ell}{\partial s \partial \bar{s}}=\frac{1}{2} \int_{\gamma} & (\operatorname{Hess}(\varphi)(\dot{u}, \dot{\bar{u}})+(A \cup \bar{A})(\dot{u}, \dot{\bar{u}}) \\
& -\bar{\partial} A(\dot{\bar{u}}, \dot{\bar{u}}, \bar{w})-2 \partial A(\dot{\bar{u}}, \bar{w}, \dot{u})-\partial A(\dot{\bar{u}}, \dot{\bar{u}}, v))
\end{aligned}
$$

with $\varphi=(\square+1)^{-1}\left(|A|^{2}\right)$.

The equations of Lemma 3.1 now read:

$$
\begin{aligned}
-\frac{D^{2}}{d t^{2}}(v)+R(\dot{u}, \dot{\bar{u}})(v) & =R(\dot{u}, \bar{w})(\dot{u})-(\uparrow \bar{\partial}) \bar{A}(\dot{u}, \dot{u}) \\
-\frac{D^{2}}{d t^{2}}(w)+R(\dot{u}, \dot{\bar{u}})(w) & =R(\dot{u}, \bar{v})(\dot{u})-2 \partial A(\dot{u}, \dot{\bar{u}})-\bar{\partial} A(\dot{\bar{u}}, \dot{\bar{u}}) .
\end{aligned}
$$

These two equations would be needed to express (41) just in terms of the KodairaSpencer tensors $A$ eliminating the vector fields $v$ and $w$.

Given a closed geodesic, it is necessary to assume that it extends in a unique, smooth way to neighboring fibers. In terms of vector fields along closed geodesics on the fibers two assumptions appear reasonable.

Assumption A. The real operator

$$
L:=-\frac{D^{2}}{d t^{2}}+R(\dot{u}, \dot{\bar{u}})
$$

on periodic vector fields along the closed geodesic $\gamma$ is invertible.

This is certainly true if the holomorphic bisectional curvature is strictly negative. That assumption, however, is too strong in our situation.

Now the formuals (42), (43) can be used to write (41) in the following way:

$$
\begin{aligned}
\frac{\partial^{2} \ell}{\partial s \partial \bar{s}}=\frac{1}{2} \int_{\gamma} & (\operatorname{Hess}(\varphi)(\dot{u}, \dot{\bar{u}})+(A \cup \bar{A})(\dot{u}, \dot{\bar{u}}) \\
& -g(L(w), w)-g(L(\bar{v}), \bar{v})-R(\dot{u}, \bar{v}, \dot{u}, \bar{w})-R(v, \dot{\bar{u}}, w, \dot{\bar{u}})) .
\end{aligned}
$$

In the last step one would eliminate the vector fields $v$ and $w$.

Assumption B. The kernel of the Jacobi operator $J$ (on Kähler manifolds)

$$
J(x):=\frac{D^{2}}{d t^{2}}(x)+R(\dot{u}, \bar{x})(\dot{u})-R(\dot{u}, \dot{\bar{u}})(x)=-L(x)+R(\dot{u}, \bar{x})(\dot{u}),
$$

operating on periodic vector fields along $\gamma$, is spanned by the tangent vector field $\dot{u}$.

\section{References}

[A-S] Axelsson, R., and G. Schumacher: Geometric approach to the Weil-Petersson symplectic form. - Comment. Math. Helv. 85, 2010, 243-257.

[E] Elstrodt, J.: Die Resolvente zum Eigenwertproblem der automorphen Formen in der hyperbolischen Ebene. Teil 1. - Math. Ann. 203, 1973, 295-330.

[F] FAY, J.: Fourier coefficients of the resolvent for a Fuchsian group. - J. Reine Angew. Math. 293/294, 1977, 143-203.

[K] Kerckhoff, S. P.: The Nielsen realization problem. - Ann. of Math. (2) 117, 1983, 235265.

[M] McMullen, C.: The moduli space of Riemann surfaces is Kähler hyperbolic. - Ann. of Math. (2) 151, 2000, 327-357. 
[SCH1] Schumacher, G.: The curvature of the Petersson-Weil metric on the moduli space of Kähler-Einstein manifolds. - In: Complex analysis and geometry, edited by V. Ancona et al., Univ. Ser. Math., Plenum Press, New York, 1993, 339-354.

[SCH2] Schumacher, G.: Asymptotics of Kähler-Einstein metrics on quasi-projective manifolds and an extension theorem on holomorphic maps. - Math. Ann. 311, 1998, 631-645.

[SCH] Schumacher, G.: Positivity of relative canonical bundles for families of canonically polarized manifolds. - arXiv:0808.3259v2.

[S-T] Schumacher, G., and St. Trapani: Weil-Petersson geometry for families of hyperbolic conical Riemann Surfaces. - Michigan Math. J. 60, 2011, 3-33.

[SIU] SiU, Y.-T.: Curvature of the Weil-Petersson metric in the moduli space of compact Kähler-Einstein manifolds of negative first Chern class. - In: Contributions to several complex variables, Hon. W. Stoll, Proc. Conf. Complex Analysis, Notre Dame/Indiana 1984, Aspects Math. E9, 1986, 261-298.

[WE] WeIL. A.: Final report on contract AF 18(603)-57. - Coll. Works, 1958.

[W] Wolf, M.: The Weil-Petersson Hessian of length on Teichmuller space. - arXiv:0902. $0203 v 1$.

[WO1] Wolpert, S.: The Fenchel-Nielsen deformation. - Ann. of Math. (2) 115, 1982, 501-528.

[WO2] Wolpert, S.: Chern forms and the Riemann tensor for the moduli space of curves. Invent. Math. 85, 1986, 119-145.

[WO3] Wolpert, S.: Geodesic length functions and the Nielsen problem. - J. Differential Geom. 25, 1987, 275-296.

[WO4] WOLPERT, S.: Convexity of geodesic-length functions: a reprise. - In: Spaces of Kleinian groups, edited by Y. Minsky and M. Sakuma, London Math. Soc. Lecture Note Ser. 329, Cambridge Univ. Press, Cambridge, 2006, 233-245.

[WO5] Wolpert, S.: Behavior of geodesic-length functions on Teichmüller space. - J. Differential Geom. 79, 2008, 277-334.

[Ye] Yeung, S.-K.: Bounded smooth strictly plurisubharmonic exhaustion functions on Teichmüller spaces - Math. Res. Lett. 10, 2003, 391-400.

Received 16 December 2010 